\title{
TRIAD system catalase, insulin, and low dose aspirin neutralize the effect of dermcidin isoform-2 and prevent cell death in acute myocardial infarction and recurrence of the disease
}

\author{
Sarbashri Bank ${ }^{1,2}$, Smarajit Maiti ${ }^{2}$, Santanu Guha ${ }^{3}$, Asru K. Sinha ${ }^{1}$ \\ ${ }^{1}$ Sinha Institute of Medical Science and Technology, Kolkata, India \\ ${ }^{2}$ Department of Biochemistry, CMT Lab, Vidyasagar University, Medinipur, India \\ ${ }^{3}$ Department of Cardiology, Calcutta Medical College and Hospital, Kolkata, India
}

Acute myocardial infarction (AMI) is a global leading cause of death in cardiovascular diseases. Stress is one of major factors predicting AMI [1]. The recurrence of AMI within a month after index hospitalization is very common in patients with cardiovascular diseases. Although the aspirin resistence may be associated with the diagnosis of a definite AMI [2], the low and precisely regulated dose of aspirin might be helpful in AMI through the inhibition of dermicidin isoform-2 (DCN-2) (Fig. $1-\mathrm{f}$ ) i.e. expulsion of platelet bound DCN-2 from AMI plasma [3].

The atherosclerotic plaques are responsible for the initiation of cell death by inhibiting the supply of $\mathrm{O}_{2}$, glucose, nutrients, and minerals to the site of injury [4]. In consequence, cells in the affected site start to die, being unable to continue core metabolic processes.

Free radical-induced oxidative stress is frequent in AMI patients. Catalase is a free-radical reductor that helps in the inhibition of platelet aggregation [5]. Different types of free radicals and $\mathrm{H}_{2} \mathrm{O}_{2}$ are generated at the time of platelet aggregation. Catalase acts as a crucial defender by destroying stress-induced free radicals, such as $\mathrm{H}_{2} \mathrm{O}_{2}$, helps in platelet aggregation inhibition, and subsequently saves the cells from death (Fig. $1-$ g, g'). The prevention of cell death is also caused by the caspase inhibition via catalase activation (Fig. 1-h) [6]. It has also been reported that $\mathrm{DCN}-2$, an envi- ronmentally stress-induced $11 \mathrm{kDa}$ protein, is involved in the prognosis of AMI. The concentration of DCN-2 protein is significantly higher in AMI circulation as compared to acute coronary syndrome patients and normal plasma [3]; it is a potent inhibitor of all forms of nitric oxide synthases (NOS) and an activator of platelet aggregation [3]. As a result, thrombus formation starts to progress, leading to cell death (Fig. $1-\mathrm{i}$ ). Low-dose-aspirin and insulin are decisively considered to be inhibitors of DCN-2 [3]. Actually, DCN-2 was able to nullify the effect of aspirin and insulin. Thus, aspirin was unable to inhibit the platelet aggregation in AMI patients. This action is related to the respective synthesis rate/kinetics and local persistence of the endogenous substances in vivo, i.e. dermcidin-2 and insulin. In a severe stressful condition, a dominating concentration of $\mathrm{DCN}-2$ may nullify aspirin and insulin effects. However, if we focus on the stepwise effects of DCN-2, i.e. binding on the platelet surface followed by the aggregation of platelets due to the inhibition of nitric oxide (NO) production, and accordingly antagonizing the effects stepwise manner, we could get substantially greater success. Hereby we show unique dosing of aspirin and insulin in a biphasic manner. The first dose of aspirin would be capable of removing the platelet-bound dermcidin from its high affinity binding sites through the production of $\mathrm{NO}$, but it was incapable of inhibiting the platelet

Address for correspondence: Prof. Asru K. Sinha, D.Sc., Sinha Institute of Medical Science and Technology, 288-Kendua main road, Baishnabghata, Garia, Kolkata-700084, India, e-mail: asruksinha@yahoo.com; Dr Sarbashri Bank, Department of Biochemistry, CMT Lab, Vidyasagar University, Medinipur, India, tel: +91 903897594 , fax: +9133 24127905, e-mail: sbank.biochem@gmail.com 


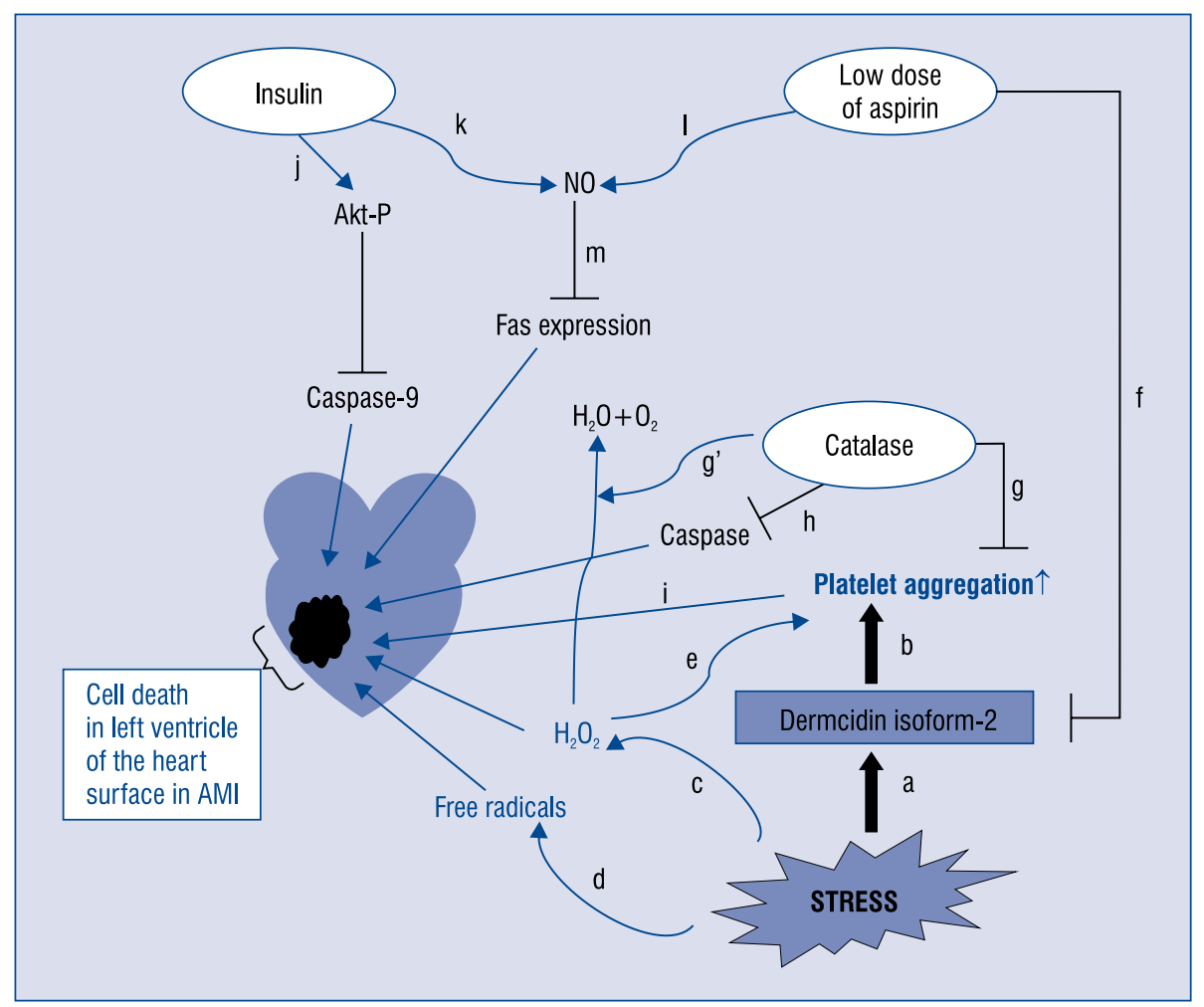

Figure 1. The figure demonstrates dermcidin isoform-2 and a stress protein-mediated platelet aggregation, followed by cell death in acute myocardial infarction (AMI) subjects. It subsequently indicates that the TRIAD system insulin, low dose aspirin and catalase would be capable of neutralizing the dermcidin effect mainly through nitric oxide (NO) pathway.

aggregation. Insulin or NO could be used instead of aspirin at first dose. After removal of dermcidin from the platelet surface, platelets become supersensitive to the aspirin and the second dose of aspirin is able to inhibit the platelet aggregation in AMI. Both of these organic (aspirin) and physiological (insulin) compounds were able to produce NO through the activation of aspirin-activated NOS [7] and insulin-activated NOS [8], respectively, and eliminated the effect of dermcidin [3]. It has also been reported before that insulin is an antithrombotic factor [9]. The high level of DCN-2 $(0.4 \mu \mathrm{M})$ in AMI subjects is the cause of massive platelet aggregation. Due to the fact that $\mathrm{DCN}-2$ is a stress-induced component, as $\mathrm{H}_{2} \mathrm{O}_{2}$ and other free radicals, it can directly activate platelet aggregation in AMI (Fig. 1 - a, b, c, d, e). Moreover, it becomes responsible for the cell death in the case of AMI (Fig. $1-$ a, b, c, d) and also causes the failure of the aspirin-effect. Specific amounts of insulin and aspirin were able to neutralize the effect of DCN-2 through the production of $\mathrm{NO}$ and as such, cell death might be inhibited by this signaling molecule through the inhibition of Fas expression (Fig. $1-\mathrm{k}, 1, \mathrm{~m}$ ). We have already reported that exact amount of aspirin or insulin was able to produce NO. Precisely regulated psychological level of NO is a very important messenger molecule that plays an instrumental role in different cells and tissues of various functions. One of the essential roles of NO is to inhibit the unnatural cell death in AMI by impairing the Fas expression (Fig. 1 — k, 1, m) [10]. Fas (Apo-1 or CD95) represents a well-known death receptor that is activated by its cognate - Fas ligand. Fas binding stimulates receptor trimerization and sequential recruitment of the adapter molecule Fas-associated death domain. The NO action impairs this signaling cascade.

On the other hand, if the availability of catalase in an AMI patient remains normal, cell death in AMI might be inhibited due to the reduction of free radicals during platelet aggregation. Therefore, by the interactive role of theTRIAD system (catalase, insulin, and aspirin), the cell death in AMI can be stopped and even the consequence of the recurrence of AMI due to the negative effect of $\mathrm{DCN}-2$ could be prevented (Fig. 1). As a result, it can also be argued that if we were able to inhibit NOS in the heart by gene silencing through double stranded RNA-interference pathway, it would be better to 
understand the efficacy of the TRIAD system on DCN-2.

In this context, our report explains the mechanism of cell death occurrence in AMI patients by the stress-induced protein DCN-2. This paper provides explanation how cell death can be prevented, and recurrence of the disease stopped, by a unique solution of maintaining catalase, insulin, and low-dose-aspirin in AMI patients.

\section{Acknowledgements}

Sarbashri Bank is a DST Inspire Fellow (DST Inspire fellow/2014/308), and would like to thank the Department of Science and Technology, Govt. of India, for providing the fellowship.

\section{Conflict of interest: None declared}

\section{References}

1. Hammoudeh AJ, Alhaddad IA. Triggers and the onset of acute myocardial infarction. Cardiol Rev, 2009; 17: 270-274.

2. Poulsen TS, Jørgensen B, Korsholm L, Licht PB, Haghfelt T, Mickley H. Prevalence of aspirin resistance in patients with an evolving acute myocardial infarction. Thromb Res, 2007; 119: 555-562.

3. Bank S, Jana P, Maiti S, Guha S, Sinha AK. Dermcidin isoform-2 induced nullification of the effect of acetyl salicylic acid in platelet aggregation in acute myocardial infarction. Sci Rep, 2014; 4: 5804.

4. Uryga AK, Bennett MR. Ageing induced vascular smooth muscle cell senescence in atherosclerosis. J Physiol, 2015; doi: 10.1113/JP270923.

5. Del Principe D, Menichelli A, De Matteis W, Di Corpo ML, Di Giulio S, Finazzi-Agro A. Hydrogen peroxide has a role in the aggregation of human platelets. FEBS Lett, 1985; 185: 142-146.

6. Murtaza I, Wang HX, Feng X et al. Down-regulation of catalase and oxidative modification of protein kinase CK2 lead to the failure of apoptosis repressor with caspase recruitment domain to inhibit cardiomyocyte hypertrophy. J Biol Chem, 2008; 283: 5996-6004.

7. Karmohapatra SK, Chakraborty K, Kahn NN, Sinha AK. The role of nitric oxide in aspirin induced thrombolysis in vitro and the purification of aspirin activated nitric oxide synthase from human blood platelets. Am J Hematol, 2007; 82: 986-995.

8. Ray U, Khan GA, Chakraborty K, Isolation and study of insulin activated nitric oxide synthase inhibitor protein in acute myocardial infarction subjects. J Thromb Thrombolysis, 2012; 33: 218-229.

9. Chakraborty K, Sinha AK. The role of insulin as an antithrombotic humoral factor. Bioessays, 2004; 26: 91-98.

10. Mannick JB, Miao XQ, Stamler JS. Nitric oxide inhibits Fasinduced apoptosis. J Biol Chem, 1997; 272: 24125-24128. 